\title{
Delayed Segmental Implant-Supported Overdenture for a Patient With Partially Resected Mandible due to Ameloblastoma
}

\author{
Sushil Kumar Kar ${ }^{1}$, Arvind Tripathi ${ }^{2}$, Ravinder Singh Bedi ${ }^{3}$
}

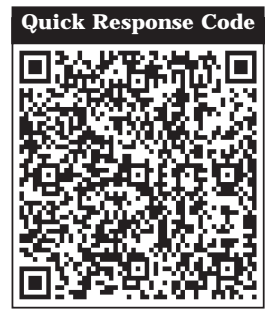

\section{doi: $10.5866 / 2015.7 .10077$}

\section{${ }^{1}$ Professor}

2Professor and Head

Department of Prosthodontics, Saraswati Dental

College and Hospital, Lucknow, Uttar Pradesh, India.

${ }^{3}$ Professor and Head

Department of Oral and Maxillofacial Surgery,

Saraswati Dental College and Hospital, Lucknow,

Uttar Pradesh, India.

\section{Article Info:}

Received: J anuary 10, 2015

Review Completed: February 9, 2015

Accepted: March 11, 2015

Available Online: April, 2015 (www.nacd.in)

(C) NAD, 2015 - All rights reserved

\section{Email for correspondence:}

drsushil_kar@yahoo.co.in

\begin{abstract}
:
Reconstruction following mandibular resection is still very challenging for prosthodontist. Mandible defects could be the consequence of ablativesurgery for malignancies, hugejaw cysts, infection and trauma. In patients with oral cancers such as ameloblastoma, the correct planning of surgery for the tumor resection as well as prosthetic rehabilitation are crucial. Resection of the mandible may compromise orofacial function and often lead to patients' psychological disorders. Despite very frequent use of mi crovascular flaps, autogenous bone grafts are still very reliable technique for mandible reconstruction. Comprehensive therapy means not only mandible reconstruction, but prosthodontic rehabilitation supported by dental implants, which can significantly improve patients' quality of life. The aim of this paper was to present a patient who had been submitted to mandible resection and reconstruction with autogenous iliac bone graft and subsequent prosthodontic rehabilitation with segmental implant supported overdenture anchoraged by ball-socket implants.
\end{abstract}

Key words: Ameloblastoma, Iliac crest graft, Mandibular reconstruction, Oral rehabilitation

\section{INTRODUCTION:}

Ameloblastoma is a aggressive odontogenic epithelial tumor. ${ }^{1}$ Radiographically, it appears as an expansile radiolucent, with thinned and perforated cortices, and it is known to cause root resorption. I ts most common site of occurrence is the mandibular molar region. Aggressive resection is the most effective method of eliminating the tumors. ${ }^{2}$ This leads to serious complications such as facial deformity, oronasal and oro-antral communications, phonetic difficulties, problems with swall owing, loss of teeth and alveolar basal processes which cause significant chewing impediments. To select a suitable reconstruction method, bone-defect should beconsidered first, foll owed by the soft tissue condition. With regard to the bone quantity and quality, the best characteristics are provided by the iliac crest. The technique of raising free iliac bone grafts is simple one, their shape matches mandible contours and dimensions and they provide enough amount of bone that is very significant for implant placement. Generally, patients experience a 
postoperative course without difficulties, and donor site complications are rare.

A definitive functional reconstruction implies prosthetic rehabilitation, which may be done with removable prosthesis which are retained by means of the existing teeth. However, apart from the limited function and discomfort of patient, removable prosthesis also causes an additional bone resorption. Rehabilitation with implant therapy is therefore potentially more acceptable and reliable for the restoration of masticatory and aesthetics functions. An ideal reconstruction is achieved with fixed dentures anchoraged by dental implants. ${ }^{3}$ If bone dimensions are not sufficient, bone augmentation or adequate implantation systems can be used. This clinical report describes the functional and esthetic reconstruction of an adult patient with segmental implant supported overdenture anchoraged by ball-socket implants following partial resection and augmentation with iliac bone grafts of mandibular ameloblastoma.

\section{Case Report:}

A 38-years-old male patient was reported with segmental reconstructed mandible. The postoperative course was uneventful. One year after the surgery, a complete graft integration with a certain degree of resorption was radiologically confirmed (Figure 1). Both clinically and radiographically, vertical and horizontal bone dimensions were found to be insufficient for placement of conventional screw implants (Figure 2). A final decision about prosthetic rehabilitation with sectional removable over dentures supported by implants was made in agreement with the patient after having been informed about all the eventual possibilities.

A good functional denture prior to placing implants is mandatory before all implant supported overdentures. So, a heat processed sectional acrylic denture was fabricated following the necessary protocols. The sectional denture was duplicated and an image guide was fabricated The patient wore the image guide for the CT scan to determine the sizes and positions of implants .On the basis of the CT scan, the implants selected were having a length of $13 \mathrm{~mm}$ with $3.5 \mathrm{~mm}$ diameter platform and $2.5 \mathrm{~mm}$ diameter Internal Hex. The image guide was then transferred into a surgical stent. The implantation procedure was performed under local anesthesia. Intraoperatively, following rising mucoperiosteal flap, the stability of bone graft was confirmed. After surgical stent adaptation, implantation was done according to the protocol for ADIN dental implants with Ball Abutments and Cap Attachments (ADIN Dental I mplant System Ltd, I srael). Three implants at the region of canine, first premolar, first molar were placed. The tops of the implants were visible and located slightly above the soft tissue. Healing screws were placed into the tops of the implants. The patient's existing denture was relieved and placed with a soft liner to prevent impingement of the implants during the postoperative healing period.

The patient was recalled after six months. A complete integration of implants was determined clinically. There were no signs of marginal boneloss around the loaded implants. The healing screws were unthreaded and removed and the Ball Abutment were screwed into the implant and tightened. A $2 \mathrm{~mm}$ height extender was used with the Ball Abutment to obtain $3 \mathrm{~mm}$ of vertical height from the top of the implant at the canine region because the implant in that location was far below the occlusal plane after bone resorption (Figure 3). The TE (Tissue Extension) also referred to as the TSI abutment, is the intermediate connector between the implant and the restoration.

Cap Attachment Transfers were placed on the Ball Abutments in the patient's mouth, and Cap Attachment Housings were placed over them. Undercuts beneath the Cap Attachment Housings are blocked out with soft utility wax to prevent the ingress of acrylic during the pick-up procedure. The base of the patient's existing denture was carefully relieved above the locations of the Cap Attachment Housings. Autopolymerizing acrylic resin (DPI India) was placed into the relieved areas of the denture and the prosthesis was carefully positioned in the patient's mouth to pick up the Cap Attachment Housings. After the material sets, voids around the housings were filled in with additional autopolymerizing acrylic, and then the tissuecontacting surfaces of the denture were smoothed and polished (Figure 4). Retentive nylon liners were pressed into the incorporated Cap Attachment Housings in the denture base and its retention was evaluated on the Ball Abutments in the patient's mouth (Figure 5). The sectional overdenture provided a group function occlusal scheme. The patient was satisfied with the functional and esthetic prosthesis, which have performed favorably for one 




Figure 1: Orthopantomograph showing complete integration of the graft after two years.

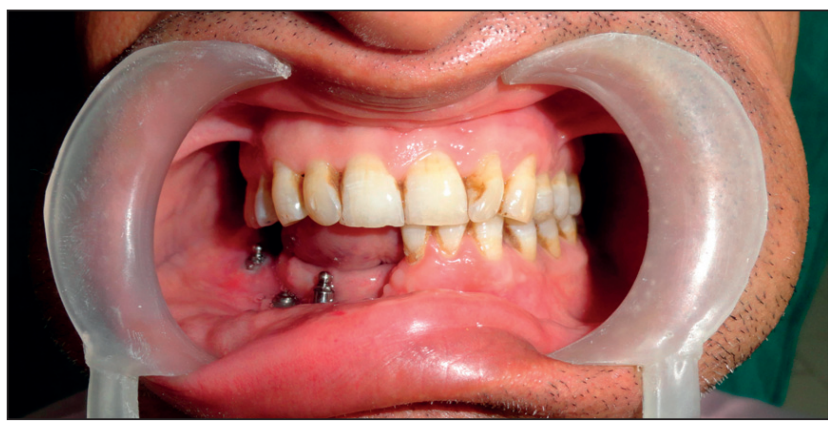

Figure 3: Ball abutments placed in the patient's mandible.

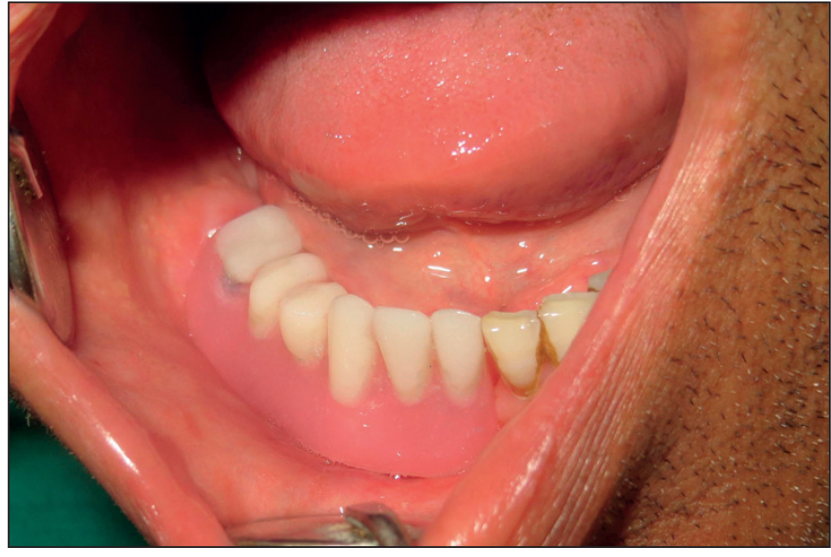

Figure 5: Prosthesis evaluated intraorally for retention.

year without any visible radiographic bone resorption (Figure 6).

\section{Discussion:}

Considering the currently available techniques for mandible reconstruction, the question arises about which cases are suitable for the reconstruction with free bone grafts and implants as method of choice. ${ }^{4}$ There are several crucial criteria for a definite decision making. Favorable tumor prognosis, good health of local tissues, and a patient's positiveattitude with realistic expectations

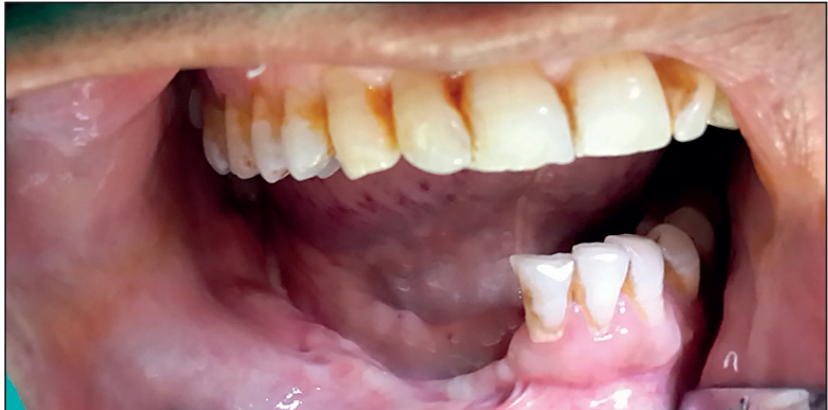

Figure 2: I ntraoral view after complete healing with deficient height and width.



Figure 4: The finished Sectional removable partial denture with Cap Attachment Housings

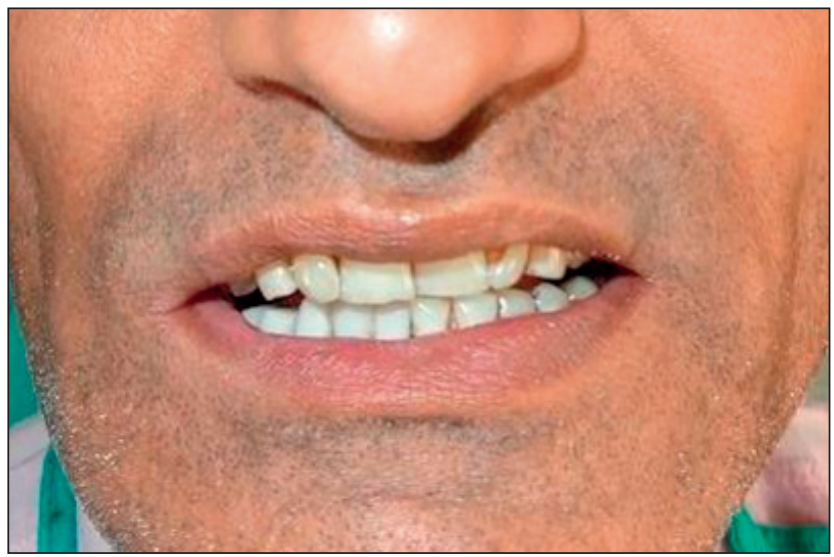

Figure 6: Extra oral view of the patient after oral rehabilitation.

are important factors for successful treatment. The majority of authors agree that the iliac crest is the best donor site, because of easy approach and possibility for taking a large amount of bone. ${ }^{5}$ One of the dilemmas is whether the reconstruction should be done simultaneously with mandible resection or subsequently. Hotz indicates an important problem of simultaneous reconstruction in cases with malignancies, because it is not possible to perform a histopathological verification of the tumor free margins. ${ }^{6}$ 
A contemporary approach to a patient definitive rehabilitation after mandible resection does not imply an anatomic reconstruction only, but also a prosthetic rehabilitation. In the past, patients were mostly rehabilitated with mobile dentures of limited functional and aesthetic values. The introduction of endosteal implants provided rehabilitation with fixed dentures showing to be more comfortable, and significantly improving both function and aesthetic. ${ }^{7}$ When considering the right timing for implants placement, there are two reasons in favor of the delayed implantation. The first is in the fact that the successful osseointegration depends on osteoblasts capable for osteogenesis, and another reason is that the simultaneous implantation is proportionally more demanding and rarely meets prosthetic requirements. ${ }^{8}$ Considering the size of the graft and the time necessary for remodeling and formation of osteogenetic potential in the presented case, the decision was made to place the implants subsequently, at least after 6 months.

Implant-retained removable overdentures are the preferred mode of treatment for some authors. ${ }^{9}$, ${ }^{10}$ When compared with a fixed prosthesis, an overdenture prosthesis allows more flexibility in denture teeth placement. Denture flanges can be contoured to reposition and support the mandibular lip. Access for oral hygiene maintenance is easier for the patient with overdenture prosthesis. The balland-socket attachment allows a full range of prosthesis motion and maximum contact between the overdentureand the mucosa during mastication. Placing the retentivesockets in the overdenture base reduces hydraulic resistance to coupling and lowers functional stresses on the implants. ${ }^{11} \mathrm{~F}$ or the denturist, the ability to decrease retention to meet specific patient needs, utilize the attachments on implants that are only relatively parallel, and replace retentive Cap Attachment Nylon Liners without the need to reline the denture make the system easy to use and maintain. Patients can eat a wider range of food items with less difficulty, and experience significantly greater confidence in conversation, social activities and intimacy than conventional denture patients. All of these factors can positively influence patient health and quality of life. ${ }^{12-14}$

\section{Conclusion:}

The reconstruction of the upper and lower jaw with iliac crest grafts in combination with dental implants, leads to satisfactory functional and aesthetic results in patients after the resection of ameloblastoma. The implant-retained overdenture represents the first choice standard of care for patients with partially resected mandibles. Prosthetic procedures and replacement of retentive sockets are relatively simple, and the ability of the patient to remove the overdenture facilitates good oral hygiene.

\section{References:}

1. Chauhan DS, Guruprasad Y, Plexiform ameloblastoma of the mandible. J Clin Imaging Sci 2011; 1:61.

2. Minichetti J C, D Amore, Schwarz E. Complete Oral Rehabilitation of a Postresection Amel oblastoma Patient: A Clinical Case Report. J Oral Implantol 2011; 37(6):735-744.

3. Goh BT, Lee S, Tideman H, Stoelinga PJ. Mandibular reconstruction in adults: a review. Int J Oral Maxillofac Surg 2008; 37(7):597-605.

4. Takushima A, Harii K, Asato H, Momosawa A, Okazaki M, Nakatsuka T. Choice of osseous and osteocutaneous flaps for mandibular reconstruction. Int J Clin Oncol 2005; 10(4):234-242.

5. Ghassemi A, Ghassemi M, Riediger D, Hilgers RD, Gerressen M. Comparison of donor-site engraftment after harvesting vascularized and nonvascularized iliac bone grafts. J Oral MaxillofacSurg 2009; 67(8):1589-1594.

6. Hotz G. Reconstruction of mandibular discontinuity defects with delayed nonvascularized free iliac crest bone grafts and endosseous implants: a clinical report. J Prosthet Dent 1996; 76(4):350-355.

7. Lundgren S, Rasmusson L, Sjöström M, Sennerby L. Simultaneous or delayed placement of titanium implants in free autogenous iliac bone grafts. Histological analysis of the bone grafttitanium interface in 10 consecutive patients. Int J Oral Maxillofac Surg 1999; 28(1):31-37.

8. Chiapasco M, Colletti G, Romeo E, Zaniboni M, Brusati R. Long term results of mandibular reconstruction with autogenous bone grafts and oral implants after tumor resection. Clin Oral Implants Res 2008; 19(10):1074-1080.

9. Chan MF, Hayter J P, Cawood JI, Howell RA. Oral rehabilitation with implant-retained prostheses following ablative surgery and reconstruction with free flaps. Int J Oral Maxillofac Implants 1997; 12(6):820-827.

10. Leung AC, Cheung LK. Dental implants in reconstructed jaws: patients' evaluation of functional and quality-of-life outcomes. Int J Oral Maxillofac Implants. 2003; 18(1):127-234.

11. Kovacs AF. I nfluence of the prosthetic restoration modality on bone loss around dental implants placed in vascularized iliac bone grafts for mandibular reconstruction. Otolaryngol Head Neck Surg 2000; 123(5):598-602.

12. FeineJ S, Carlsson GE, Awad MA,et al. The McGill consensus statement on overdentures. Montreal, Quebec, Canada. May 24-25, 2002. Int J Prosthodont 2002; 15:413-414.

13. Firtell DN, Curtis TA. Removable partial denture design for the mandibular resection patient. J Prosthet Dent 1982; 48:437-443.

14. Kratochvil FJ. Defects with mandibular continuity. In: Beumer J III, Curtis TA, Firtell DN, eds. Maxillofacial Rehabilitation: Prosthodontic and Surgical Considerations. St Louis: Mosby, 1979. 